Article

\title{
An Efficient Agrobacterium-Mediated Transformation System for Poplar
}

\author{
Ali Movahedi ${ }^{1}$, Jiaxin Zhang ${ }^{1}$, Rasoul Amirian ${ }^{2}$ and Qiang Zhuge ${ }^{1, *}$ \\ 1 The Cooperative Innovation Center of Southern Modern Forestry, Nanjing Forestry University, \\ Nanjing 210037, China; E-Mails: ali664699@gmail.com (A.M.); zhangjiaxin60633@163.com (J.Z.) \\ 2 Department of Genomics, Agricultural Biotechnology Research Institute, \\ Central Region of Iran (ABRICI), Najafabad Road, Isfahan 85135-487, Iran; \\ E-Mail: rasool_amirian@yahoo.com \\ * Author to whom correspondence should be addressed; E-Mail: qzhuge@njfu.edu.cn; \\ Tel./Fax: +86-25-8542-8701.
}

Received: 1 March 2014; in revised form: 3 June 2014 / Accepted: 4 June 2014 /

Published: 13 June 2014

\begin{abstract}
Poplar is a model system for the regeneration and genetic transformation of woody plants. To shorten the time required for studies of transgenic poplar, efforts have been made to optimize transformation methods that use Agrobacterium tumefaciens. In this study, an Agrobacterium infective suspension was treated at $4{ }^{\circ} \mathrm{C}$ for at least $10 \mathrm{~h}$ before infecting explants. By transforming the Populus hybrid clone "Nanlin895" (Populus deltoides $\times$ P. euramericana) with Agrobacterium harboring the PBI121:CarNAC6 binary vector, we showed that the transformation efficiency was improved significantly by multiple independent factors, including an Agrobacterium infective suspension with an $\mathrm{OD}_{600}$ of 0.7, an Agrobacterium infection for $120 \mathrm{~min}$, an Agrobacterium infective suspension at a $\mathrm{pH}$ of 5.0, an acetosyringone concentration of $200 \mu \mathrm{M}$, a cocultivation at $28{ }^{\circ} \mathrm{C}$, a cocultivation for $72 \mathrm{~h}$ and a sucrose concentration of $30 \mathrm{~g} / \mathrm{L}$ in the cocultivation medium. We also showed that preculture of wounded leaf explants for two days increased the regeneration rate. The integration of the desired gene into transgenic poplars was detected using selective medium containing kanamycin, followed by southern blot analysis. The expression of the transgene in the transgenic lines was confirmed by northern blot analysis.
\end{abstract}

Keywords: transformation system; Populus; Agrobacterium-mediated; efficient 


\section{Introduction}

Poplar is a versatile tree species that is highly amenable to vegetative propagation, has a rapid growth rate and is a good model system for the transformation of woody plant species. These characteristics have led to its widespread use in the pulp, paper and cosmetics industries and in both highland and lowland reforestation [1-4]. Furthermore, poplar is a forest tree species, with a rather long generation cycle (seven to 10 years or more). That is why researchers usually use materials from the initial generation for the analysis of transgenic plants of forest trees, with just several months' generation cycles. Efforts to optimize regeneration and propagation methods that use leaves, petioles, internodes, stems, roots and sprouts to improve poplar through the overexpression of transgenes conferring resistance to biotic and abiotic stresses or other traits have increased markedly over the past decade [2,3,5,6]. Transformation methods using leaf segments are the most commonly used [7]. Attempts to improve poplar transformation mediated by Agrobacterium tumefaciens have focused on a variety of parameters, including different Agrobacterium strains and culture densities, various acetosyringone (AS) concentrations and other factors[1,8-11].

The expression of high-copy number transgenes in transgenic plants may be more or less than only a single copy of transgene, and also, sometimes, this is good for molecular analysis and genetic engineering. Furthermore, Husaini [12] reported that strawberry transgenic plants with a high transgene copy number (four copies or more) are very good for molecular analysis and genetic engineering. Moreover, Bartlett et al. [13] reported that an improved method in barely using Agrobacterium, with a high copy of transgenes, is quite good for molecular analysis and genetic engineering. The benefits of improved transformation methods include the insertion of intact full-length cDNAs into the plant genome, a reduction in the number of undesirable mutations and an increased transgene copy number [4]. For these reasons, many efforts have been made to improve Agrobacterium-mediated transformation efficiency [14,15]. The CarNAC6 gene from chickpea encodes a 308-amino-acid, plant-specific regulatory transcriptional factor belonging to the NAC (NAM, ATAF1,2, and CUC2) domain protein family that increases plant response reactions to biotic and abiotic stresses and plays an important role in plant development. Homologues of the CarNAC6 gene have been identified in Arabidopsis and rice [16-20]. In this study, we transformed the poplar hybrid clone "Nanlin895" (Populus deltoides $\times$ P. euramericana "Nanlin895") with the PBI121:CarNAC6 binary vector to improve Agrobacterium-mediated transformation efficiency. In addition, we attempted to optimize MS culture medium supplemented with $N$-6-benzyladenine (6-BA) and thidiazuron (TDZ) hormones to minimize lateral shoot development. Other factors affecting transformation efficiency evaluated in this study included the preculture of wounded explants, Agrobacterium infective suspension concentration, Agrobacterium infection duration, Agrobacterium infective suspension $\mathrm{pH}$, acetosyringone (AS) concentration, cold treatment of an Agrobacterium infective suspension, cocultivation incubation temperature and duration and sucrose concentration in cocultivation medium. All of these factors were treated independently in related experiments independent of the age of the explants. In this study, the transformation efficiency is expressed as the percentage of independently transformed explants relative to the total number of explants. 


\section{Results and Discussion}

\subsection{Optimization of Poplar Regeneration}

An overview of the steps used in the Agrobacterium-mediated transformation of Populus species is presented in Figure 1. The poplar hybrid clone "Nanlin895" (Populus deltoides $\times$ P. euramericana "Nanlin895") was cultured in medium containing various concentrations of 6-BA and TDZ (Table 1). Little or no regeneration occurs in the absence of 6-BA. Medium containing $0.5 \mathrm{mg} / \mathrm{L}$ 6-BA was used to achieve the maximum regeneration rate ( $85 \%$ to $90 \%)$. Increases in non-specific poplar regeneration resulted in higher numbers of lateral shoots relative to the numbers of main shoots. TDZ, an inducer of shoot regeneration, was used to increase the specific regeneration of main shoots. The proportion of main shoots to lateral shoots was increased by TDZ concentrations of 0.002 and $0.004 \mathrm{mg} / \mathrm{L}$, but was decreased by concentrations of 0.008 and $1.0 \mathrm{mg} / \mathrm{L}$. The optimal regeneration medium contained $0.5 \mathrm{mg} / \mathrm{L}$ 6-BA and $0.004 \mathrm{mg} / \mathrm{L} \mathrm{TDZ}$ to maximize the frequency of main shoot regeneration. The rates of shoot elongation (Figure $2 \mathrm{~d}$ ) and root regeneration (Figure $2 \mathrm{e}$ ) were enhanced by up to $100 \%$ when using the optimized medium. As shown in Figures $2 \mathrm{a}$ and 3, the preculture of explants for two days caused higher transformation efficiencies than preculture for one and three days. Therefore, explants precultured for two days were used subsequently for transformation with Agrobacterium.

Figure 1. An overview the steps for Agrobacterium-mediated transformation in Populus species.

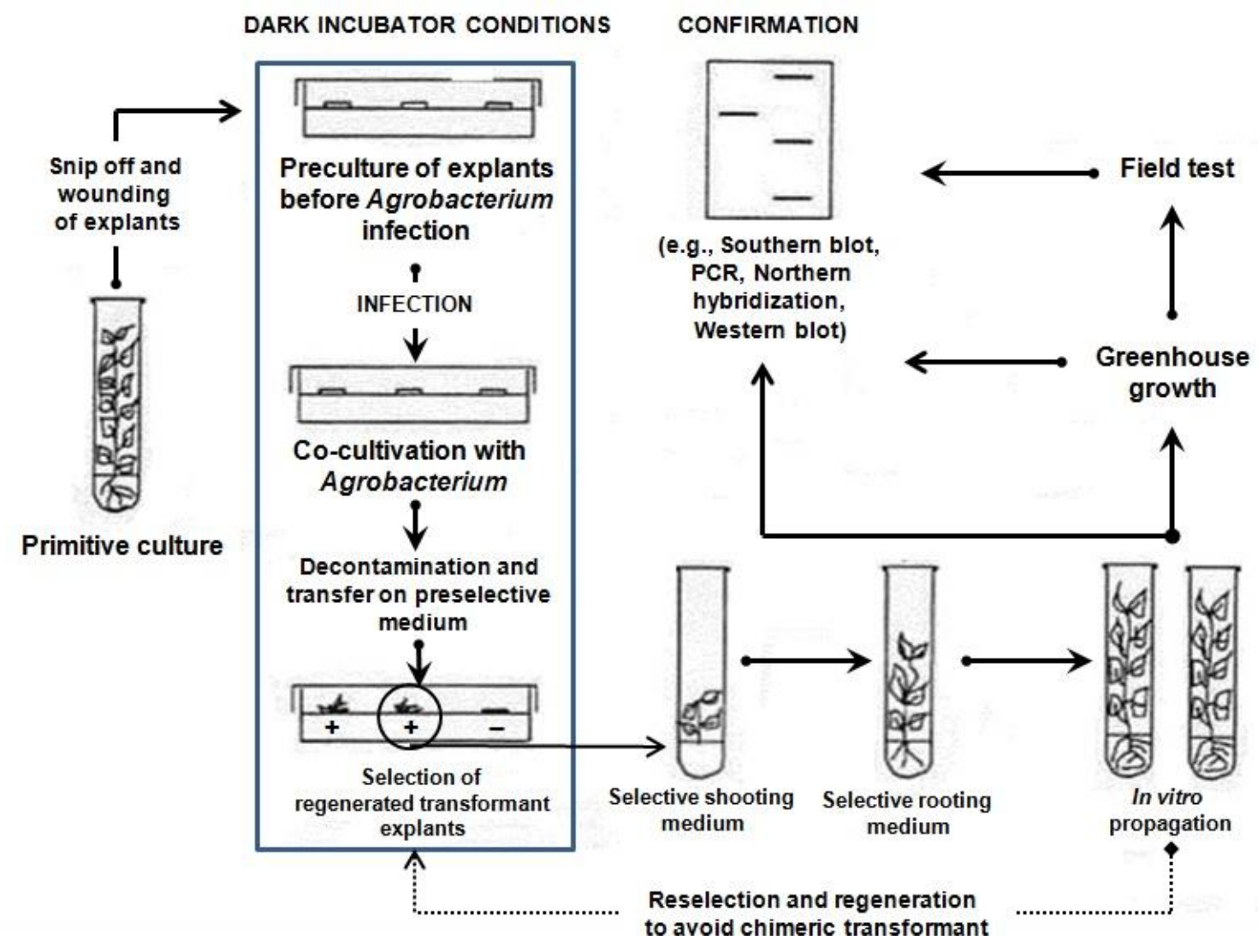


Figure 2. (a) Cocultivation of poplar leaf discs with Agrobacterium on MS medium; (b) Regenerated putative poplar transformants on MS selection medium that were generated without the optimization of transformation efficiency; (c) Regenerated putative poplar transformants on MS selection medium generated with the optimization of transformation efficiency; (d) MS shoot elongation medium; (e) Half MS rooting medium. Scale bar $=1 \mathrm{~cm}$.
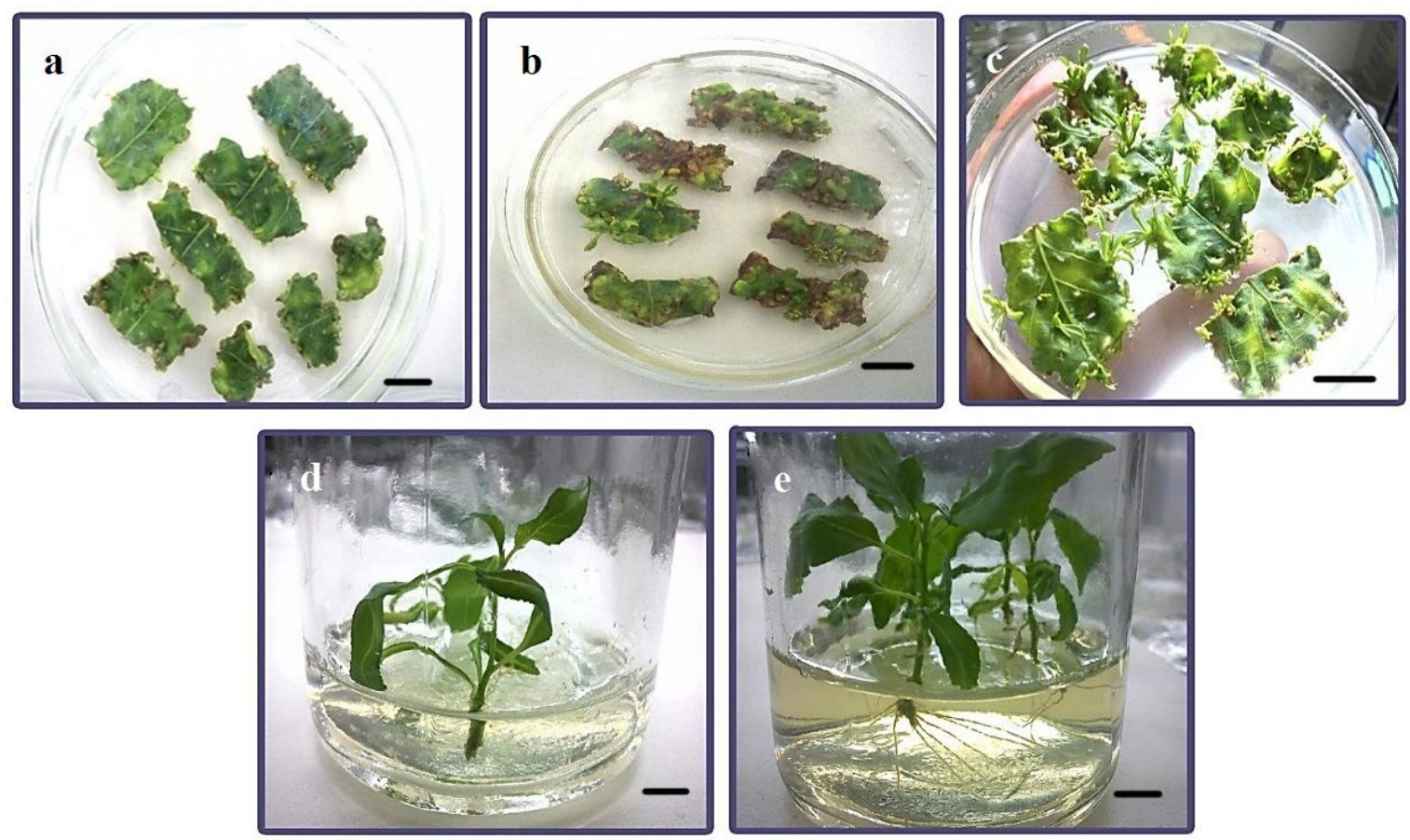

Table 1. The effect of N-6-benzyladenine (6-BA) and thidiazuron (TDZ) on the main shoot and total shoot regeneration (\%).

\begin{tabular}{|c|c|c|c|c|}
\hline $\begin{array}{l}\text { Medium } \\
\text { Number }\end{array}$ & $\begin{array}{c}\text { 6-BA } \\
(\mathrm{mg} / \mathrm{L})\end{array}$ & $\begin{array}{c}\text { TDZ } \\
(\mathrm{mg} / \mathrm{L})\end{array}$ & $\begin{array}{c}\text { Mean Number of Main Shoots per } \\
\text { Medium (Number of Main Shoots in } \\
\text { Each Medium/Number of Explants in } \\
\text { Each Medium) }\end{array}$ & $\begin{array}{c}\text { Total Shoot } \\
\text { Regeneration in } \\
\text { Each Medium (\%) }\end{array}$ \\
\hline 1 & 1.00 & 0.000 & 3.10 & 89.0 \\
\hline 2 & 1.00 & 0.002 & 3.50 & 93.0 \\
\hline 3 & 1.00 & 0.004 & 4.80 & 94.0 \\
\hline 4 & 1.00 & 0.008 & 3.30 & 89.0 \\
\hline 5 & 1.00 & 0.010 & 2.90 & 97.0 \\
\hline 6 & 0.80 & 0.000 & 38.8 & 85.0 \\
\hline 7 & 0.80 & 0.002 & 43.0 & 82.0 \\
\hline 8 & 0.80 & 0.004 & 45.8 & 78.0 \\
\hline 9 & 0.80 & 0.008 & 40.7 & 80.0 \\
\hline 10 & 0.80 & 0.010 & 30.8 & 79.0 \\
\hline 11 & 0.50 & 0.000 & 56.8 & 80.0 \\
\hline 12 & 0.50 & 0.002 & 65.2 & 75.0 \\
\hline
\end{tabular}


Table 1. Cont.

\begin{tabular}{|c|c|c|c|c|}
\hline $\begin{array}{l}\text { Medium } \\
\text { Number }\end{array}$ & $\begin{array}{c}6-\mathrm{BA} \\
(\mathrm{mg} / \mathrm{L})\end{array}$ & $\begin{array}{c}\text { TDZ } \\
(\mathrm{mg} / \mathrm{L})\end{array}$ & $\begin{array}{c}\text { Mean Number of Main Shoots per } \\
\text { Medium (Number of Main Shoots in } \\
\text { Each Medium/Number of Explants in } \\
\text { Each Medium) }\end{array}$ & $\begin{array}{c}\text { Total Shoot } \\
\text { Regeneration in } \\
\text { Each Medium (\%) }\end{array}$ \\
\hline 13 & 0.50 & 0.004 & 69.5 & 73.0 \\
\hline 14 & 0.50 & 0.008 & 60.5 & 76.0 \\
\hline 15 & 0.50 & 0.010 & 44.4 & 70.0 \\
\hline 16 & 0.20 & 0.000 & 8.90 & 37.0 \\
\hline 17 & 0.20 & 0.002 & 11.0 & 29.0 \\
\hline 18 & 0.20 & 0.004 & 14.0 & 35.0 \\
\hline 19 & 0.20 & 0.008 & 9.00 & 32.0 \\
\hline 20 & 0.20 & 0.010 & 6.13 & 26.0 \\
\hline 21 & 0.00 & 0.000 & 0.30 & 6.00 \\
\hline 22 & 0.00 & 0.002 & 0.50 & 9.00 \\
\hline 23 & 0.00 & 0.004 & 0.80 & 13.0 \\
\hline 24 & 0.00 & 0.008 & 0.50 & 10.0 \\
\hline 25 & 0.00 & 0.010 & 0.10 & 8.00 \\
\hline
\end{tabular}

Figure 3. Precultured, wounded poplar explants before induction by Agrobacterium.

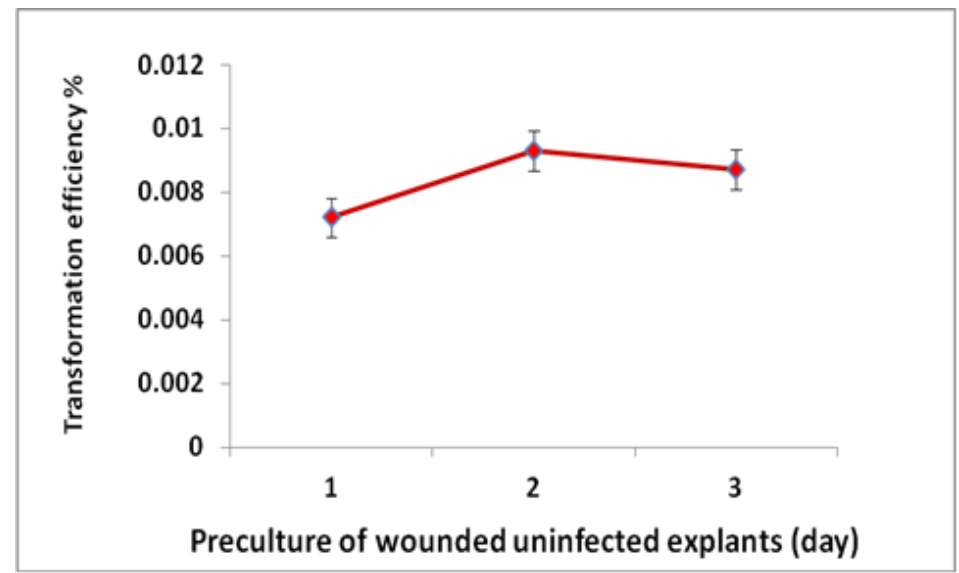

\subsection{Improvement of Agrobacterium-Mediated Transformation Efficiency}

The highest transformation efficiency was obtained from precultured explants immersed in an Agrobacterium infective suspension with an $\mathrm{OD}_{600}$ of 0.7 . The use of Agrobacterium infective suspensions with higher densities resulted in decreased transformation efficiencies, due to increased infection (Figure 4a). We examined the effect of the duration of immersion in Agrobacterium infective suspensions on the transformation efficiency. The highest transformation efficiencies were obtained from immersion for 120 and $150 \mathrm{~min}$ and were not significantly different (Figure $4 \mathrm{~b}$ ). In addition, we examined the effect of the $\mathrm{pH}$ of the Agrobacterium infective suspension on the transformation efficiency. Low $\mathrm{pHs}$ increased the efficiency, but higher $\mathrm{pHs}$ decreased the transformation efficiency, due to decreased Agrobacterium growth (Figure 4c). Increasing the AS concentration in the Agrobacterium infective suspension to as high as $200 \mu \mathrm{M}$ improved the transformation efficiency, 
probably by increasing the ability of the Agrobacterium to detect wounded plant cells. The transformation efficiencies obtained with the different AS concentrations were significantly different (Figure 4d).

We developed a cold pre-treatment of the Agrobacterium infective suspension inspired by the freeze-thaw method [21]. The transformation efficiency was increased approximately $7 \%$ by pre-treatment of the suspension at $4{ }^{\circ} \mathrm{C}$ for at least $24 \mathrm{~h}$ (Figure $4 \mathrm{e}$ ). The effect of the cold pre-treatment of the Agrobacterium infective suspension on the transformation efficiency was clearly demonstrated by the differences between cultures on plates for which the suspension had not received (Figure $2 b$ ) or had received (Figure 2c) the cold pre-treatment. The temperature at which the Agrobacterium infective suspension and the explants were co-cultivated also affected the transformation efficiency. Cocultivation at $28{ }^{\circ} \mathrm{C}$ produced the highest transformation efficiency, with significant differences in efficiency between the temperatures tested (Figure 4f). The duration of the cocultivation of the Agrobacterium infective suspension and explants was an important factor in the transformation efficiency. Cocultivation for up to $72 \mathrm{~h}$ yielded the highest transformation efficiency, with significant differences between incubation temperatures (Figure 4g).

Figure 4. The effects of various factors on Agrobacterium-mediated transformation efficiency: (a) Agrobacterium concentration; (b) Agrobacterium infection duration; (c) Agrobacterium suspension $\mathrm{pH}$; (d) acetosyringone (AS) concentration; (e) cold pre-treatment; (f) cocultivation temperature; (g) cocultivation duration; (h) cocultivation medium sucrose concentration.
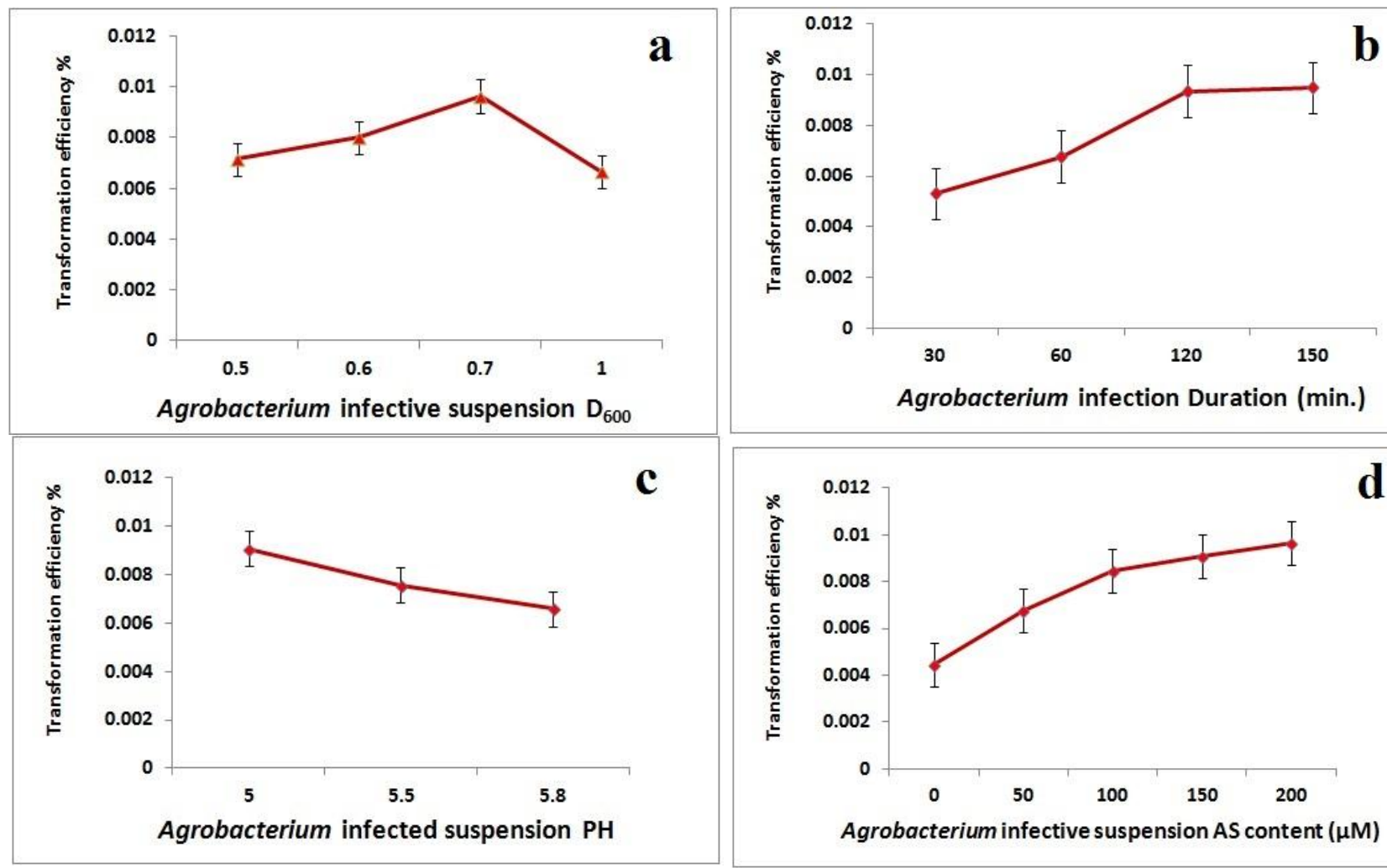

Agrobacterium infection Duration (min.)

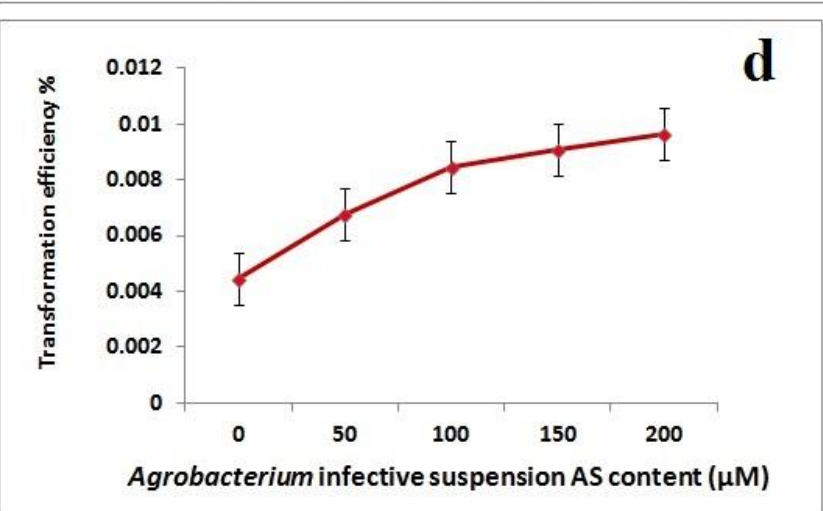


Figure 4. Cont.

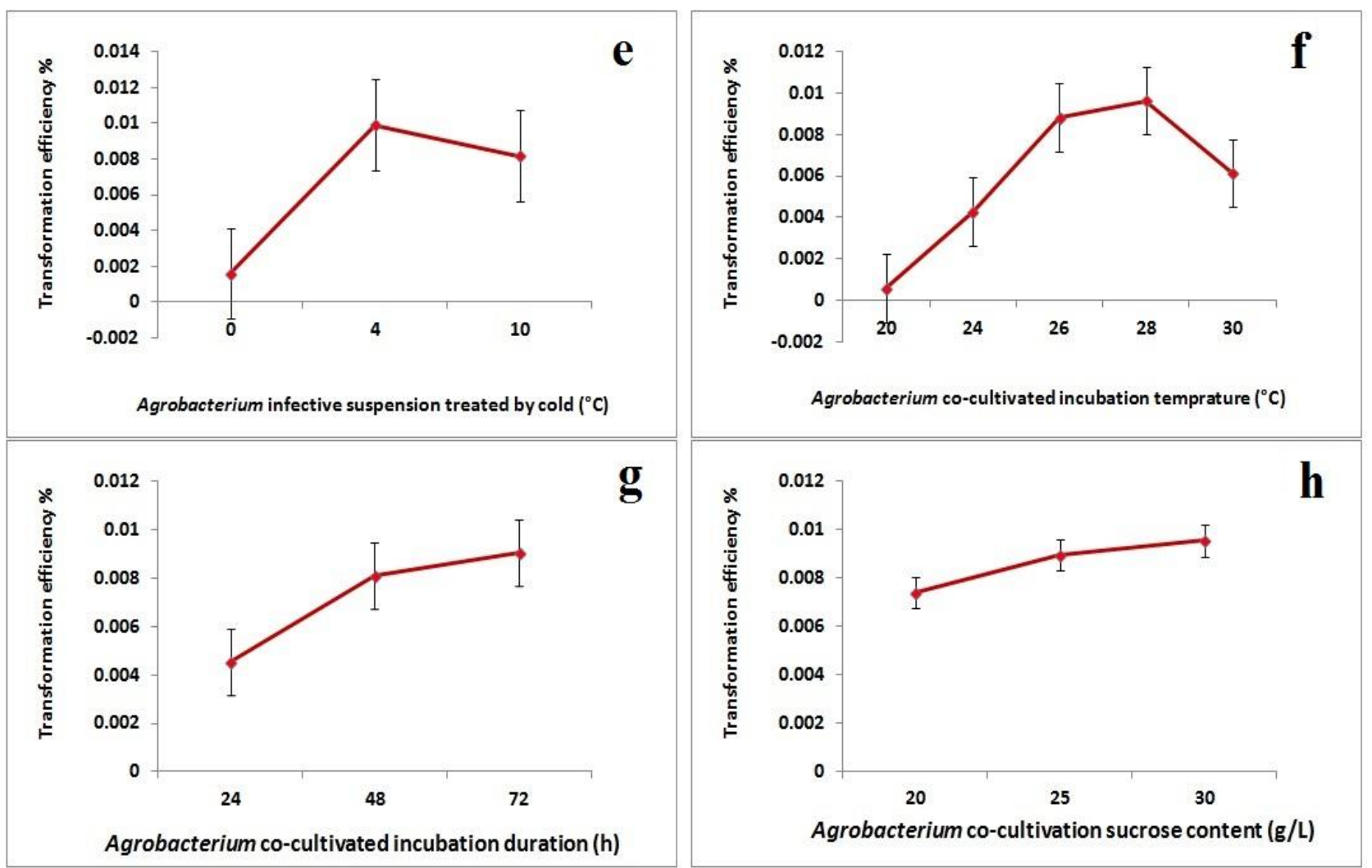

Sucrose is a required carbon source for the growth of Agrobacterium. The effects of various sucrose concentrations on transformation efficiency during the cocultivation of the Agrobacterium infective suspension and explants were significantly different, with the highest transformation efficiency obtained using a sucrose concentration of $30 \mathrm{~g} / \mathrm{L}$ (Figure $4 \mathrm{~h}$ ).

\subsection{Southern and Northern Blot Analyses}

Total genomic DNA isolated from transgenic plants was digested with SalI, which has no restriction site in the T-DNA, and with EcoRI, for which one restriction site is located downstream of the NOS (nopaline synthase) terminator. Southern blot analysis showed that 1-2 transgene copies were present in each of the three lines generated in procedures that did not show improved transformation efficiency (Figure 5a) and that 3-4 transgene copies were present in each of the three lines generated using procedures resulting in improved transformation efficiency (Figure $5 \mathrm{~b}$ ).

The detection of mRNAs of $0.5-0.8 \mathrm{kbp}$ by northern blot analysis confirmed that the CarNAC6 transgene was expressed in three transgenic poplar lines generated without improved transformation efficiency (Figure 6a) and in three transgenic poplar lines generated with improved transformation efficiency (Figure 6b). The transgene expression levels in the two line types were clearly different. Supplementation of media with hormones has been investigated to determine optimal conditions for shoot regeneration with a minimum of lateral shoots and for root regeneration. High concentrations of TDZ can result in the regeneration of a high percentage of adventitious shoots [22]. Ferreira et al. [2] reported that the regeneration of adventitious shoots from organogenic nodules of $P$. euphratica leaf explants was achieved using a range of $\alpha$-naphthaleneacetic acid (NAA) and 6-BA concentrations. 
Various concentrations of indoleacetic acid (IAA) were also shown to affect the frequency of regeneration from poplar explants [23]. Confalonieri et al. [1] reported positive effects of BA and NAA on shoot regeneration in P. nigra. Various media are required for shoot and root regeneration of different genotypes and are supplemented with various levels of indole-3-butyric acid (IBA) to induce rooting from aspen leaves and with zeatin to induce shooting [24]. The use of specific concentrations of shoot-inducing hormones, such as 6-BA, can increase the mean number of shoots formed per explant following regeneration, but typically causes decreased numbers of main shoots following regeneration [4]. Recently, the number of reports of successful Agrobacterium-mediated transformation of various plant species has increased markedly [22]. Developments in tissue culture methods and improved transformation techniques have led to the use of suitable Agrobacterium strains [25]. The development of techniques that increase transformation efficiency or the pathogenicity of Agrobacterium has been the subject of many studies. The use of appropriate AS concentrations during cocultivation has been shown to increase transformation efficiencies. Moreover, the effects of the Agrobacterium concentration and infection time on transformation efficiency have been demonstrated by Han et al. [4].

Figure 5. (a) Southern blot analysis of transgenic poplar lines generated without improved transformation efficiency. Genomic DNA extracted from three transgenic poplar lines was digested separately with SalI and EcoRI and blotted onto a nylon membrane. The blots were probed with an 868-bp PCR fragment generated from CarNAC6 cDNA. Lane 1: marker ( $\Lambda$ HindIII). Lane 2: PBI121:CarNAC6 plasmid digested with EcoRI; Lanes 3-5: genomic DNA extracted from three transgenic poplar lines digested with SalI; Lanes 6-8: the same genomic DNA digested with EcoRI; Lane 9: wild-type (WT) poplar; (b) Southern blot analysis of transgenic poplar lines generated with improved transformation efficiency.
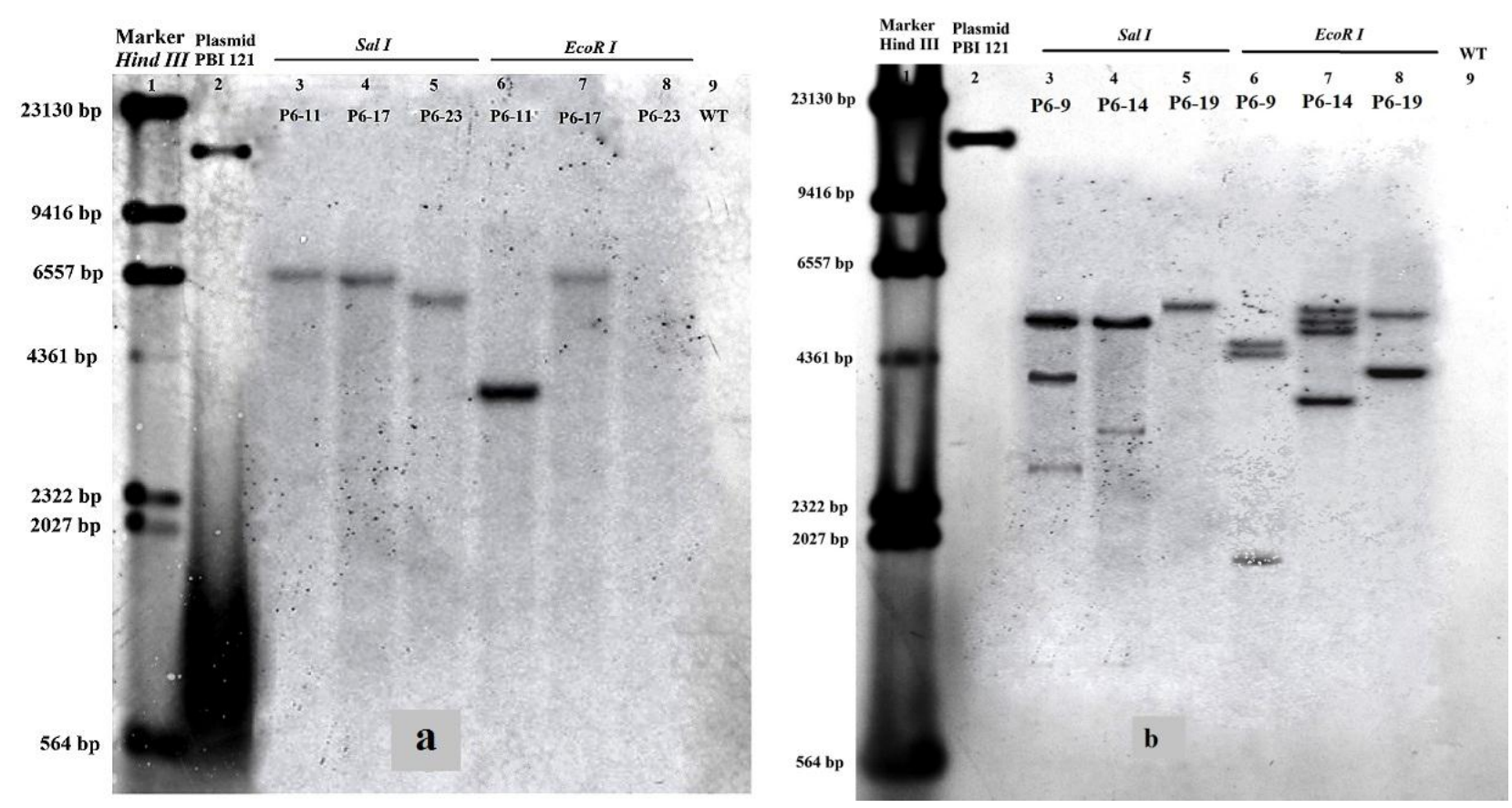
Figure 6. (a) Northern blot analysis of transgenic poplar lines generated without improved transformation efficiency. Total RNA (ribonucleic acid) was separated on a formaldehyde gel and blotted onto a nylon membrane after staining with ethidium bromide. A 433-bp fragment of CarNAC6 cDNA generated by PCR (polymerase chain reaction) was used as a probe. CarNAC6 gene expression $(0.5-0.8-\mathrm{kb}$ band) in transgenic poplar lines. Lane 1: marker ( $\lambda$ HindIII); Lane 2: wild-type (WT) poplar; Lanes 3-5: total RNA extracted from three transgenic poplar lines; (b) Northern blot analysis of transgenic poplar lines generated with improved transformation efficiency.
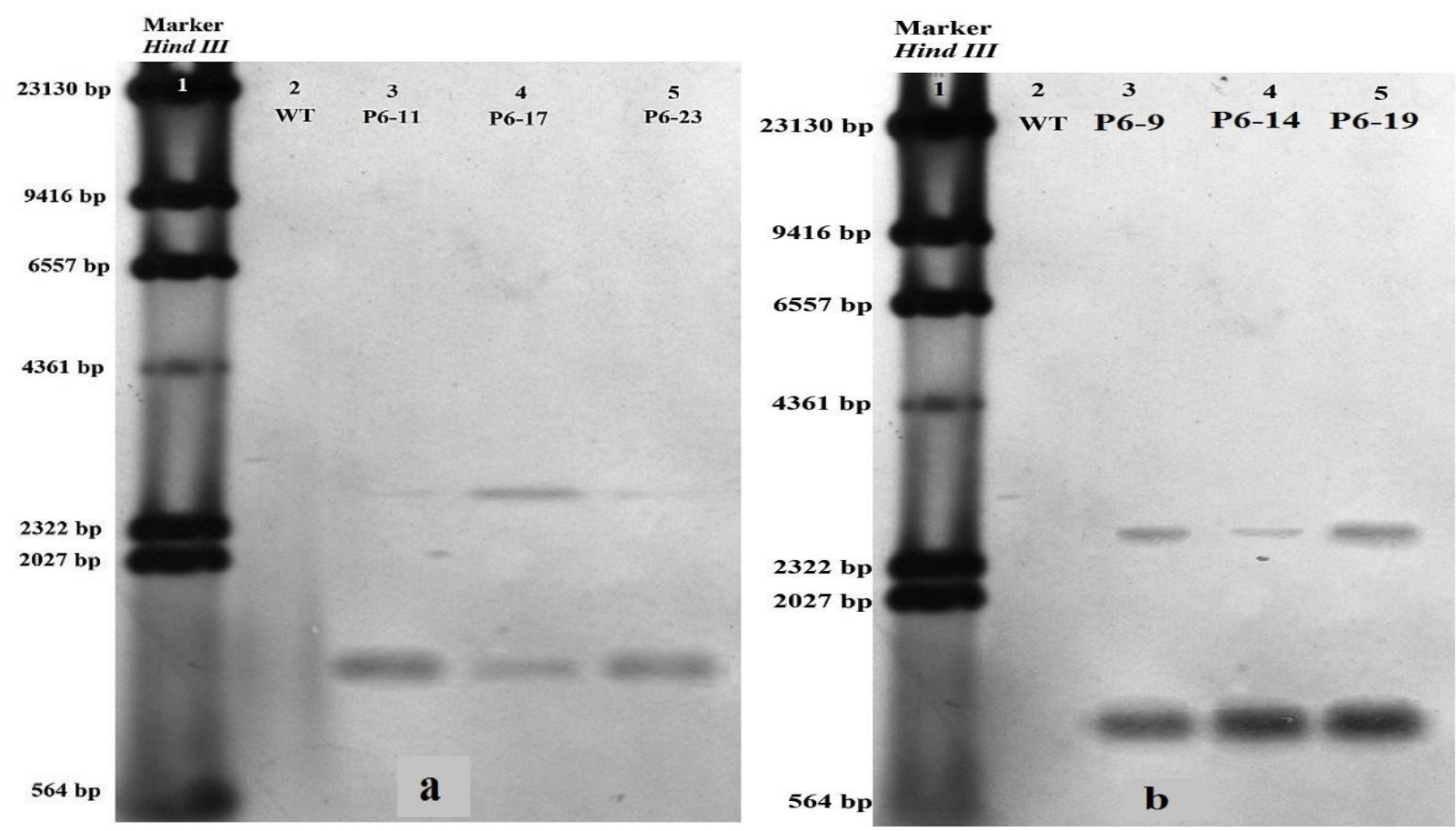

\section{Experimental Section}

\subsection{Plant Materials and Agrobacterium Strain}

Wild-type (WT) poplar leaves were collected from young $P$. deltoides $\times P$. euramericana "Nanlin895" seedlings to propagate 25 lines. The binary vector, PBI121:CARNAC6, was generated at the State Key Laboratory of Crop Genetics and Germplasm Enhancement of the National Center for Soybean Improvement at Nanjing Agricultural University, China [26]. The pBI121 plasmid was digested with $X b a \mathrm{I}$ and $B a m \mathrm{HI}$ restriction enzymes, and a 924-bp nucleotide DNA fragment containing a full-length cDNA of the chickpea CARNAC6 gene was inserted. CARNAC6 expression was driven by the CaMV $35 S$ promoter. The plasmid was introduced into the Agrobacterium tumefaciens strain, EHA105, using the freeze-thaw method [21].

\subsection{Optimization of Regeneration Medium for Poplar}

Murashige and Skoog (MS) medium [27] was supplemented with 6-benzylaminopurine (6-BA) at five concentrations $(1.0,0.8,0.5,0.2$ and $0 \mathrm{mg} / \mathrm{L})$ in combination with thidiazuron (TDZ) at five concentrations $(0,0.002,0.004,0.008$ and $0.01 \mathrm{mg} / \mathrm{L})$ to determine the optimal combination for 
minimal lateral shoot development. Immature leaves were excised as $1-\mathrm{cm}^{2}$ discs and cultured on MS medium supplemented with the two hormones. The medium was refreshed at 2-week intervals. Regenerated shoots of $2 \mathrm{~cm}$ in length were cut and rooted on sterile half-strength MS medium containing $25 \mathrm{~g} / \mathrm{L}$ sucrose and $6 \mathrm{~g} / \mathrm{L}$ agar adjusted to $\mathrm{pH} 5.8$ with no added hormones. After 6-8 weeks, the roots were examined, and leaves were collected from well-developed plantlets for gene transformation.

\subsection{Agrobacterium-Mediated Transformation}

Young leaves were cut into $0.5 \times 0.5-\mathrm{cm}$ sections and precultured on half-strength MS medium supplemented with 6 -BA $(0.5 \mathrm{mg} / \mathrm{L})$ and TDZ $(0.004 \mathrm{mg} / \mathrm{L})$ for 1,2 or 3 days. This medium was tested on 37-40 lines for each variable, with four replicates. Agrobacterium containing the binary vector, PBI121:CARNAC6, was inoculated into Luria-Bertani (LB) medium supplemented with $50 \mathrm{mg} / \mathrm{L}$ rifampicin (Rif) and $50 \mathrm{mg} / \mathrm{L}$ kanamycin (Kan) and cultured in an incubator without light at $28^{\circ} \mathrm{C}$ for $72 \mathrm{~h}[28]$.

A single Agrobacterium colony was inoculated into $5 \mathrm{~mL}$ of LB medium containing $50 \mathrm{mg} / \mathrm{L}$ Rif and $50 \mathrm{mg} / \mathrm{L}$ Kan and incubated at $28{ }^{\circ} \mathrm{C}$ for $24 \mathrm{~h}$ with constant agitation $(220 \mathrm{rpm})$. An additional $50 \mathrm{~mL}$ of LB were added and growth was continued overnight under the same conditions until an $\mathrm{OD}_{600}$ of 2.0 was reached. The culture was centrifuged at $3000 \mathrm{rpm}$ for $10 \mathrm{~min}$. The pellet was diluted in liquid MS medium with 5\% sucrose to prepare an Agrobacterium-infective suspension. All Agrobacterium experiments were carried out independently using 20-25 precultured explants in triplicate.

The precultured explants were immersed in various concentrations of Agrobacterium-infective suspensions $\left(\mathrm{OD}_{600}\right.$ values of $0.5,0.6,0.7$ or 1.0$)$. The precultured explants were also cold treated independently at 0,4 or $10{ }^{\circ} \mathrm{C}$. Other parameters tested independently for their effects on transformation efficiency included the duration of the cocultivation of the Agrobacterium-infective suspension with explants $\left(30,60,120\right.$ or $150 \mathrm{~min}$ at $28{ }^{\circ} \mathrm{C}$ with constant agitation), the AS concentration $(0,50,100,150$ or $200 \mu \mathrm{M})$ and the $\mathrm{pH}$ of the Agrobacterium infection suspension ( $\mathrm{pH} 5.0,5.5$ or 5.8). Wounded leaf discs were dried on sterile paper to remove surface water. The infected explants were co-cultivated in a dark incubator at various temperatures $(20,24,26,28$ or $30{ }^{\circ} \mathrm{C}$ ) and for different durations $(24,48$ or $72 \mathrm{~h}$ ) on semi-liquid MS induction medium containing $0.5 \mathrm{mg} / \mathrm{L}$ 6-BA, $0.004 \mathrm{mg} / \mathrm{L} \mathrm{TDZ}, 3 \mathrm{~g} / \mathrm{L}$ agar, various sucrose concentrations $(20,25$ or $30 \mathrm{~g} / \mathrm{L})$ and $200 \mu \mathrm{M}$ AS to induce the expression of the Agrobacterium Vir genes [29]. The parameters used for control transformations were the preculture of explants for 1 day, an Agrobacterium culture $\mathrm{OD}_{600}$ of $0.5,10{ }^{\circ} \mathrm{C}$ cold pre-treatment, $30 \mathrm{~min}$ of Agrobacterium cocultivation, $100 \mu \mathrm{M} \mathrm{AS}$, an Agrobacterium-infective suspension at $\mathrm{pH} 5.8$, cocultivation in darkness at $28{ }^{\circ} \mathrm{C}$ for $48 \mathrm{~h}$ and $25 \mathrm{~g} / \mathrm{L}$ sucrose in MS induction medium. Putative transformed explants were transferred to MS selection medium supplemented with $0.5 \mathrm{mg} / \mathrm{L}$ 6-BA, $0.004 \mathrm{mg} / \mathrm{L} \mathrm{TDZ}, 6 \mathrm{~g} / \mathrm{L}$ agar, $25 \mathrm{~g} / \mathrm{L}$ sucrose, $400 \mathrm{mg} / \mathrm{L}$ cefotaxime (Cef) and $50 \mathrm{mg} / \mathrm{L}$ kanamycin (Kan) at $\mathrm{pH} 5.8$ and cultured under 16-h/8-h light/dark conditions at $23 \pm 1{ }^{\circ} \mathrm{C}$ in a phytotron. Selected regenerated shoots were separated and transferred to MS shoot elongation medium supplemented with $0.25 \mathrm{mg} / \mathrm{L}$ 6-BA, $0.002 \mathrm{mg} / \mathrm{L} \mathrm{TDZ,} 6 \mathrm{mg} / \mathrm{L}$ agar, $25 \mathrm{mg} / \mathrm{L}$ sucrose and $300 \mathrm{mg} / \mathrm{L}$ Cef. Shoots of approximately $2 \mathrm{~cm}$ in length were then transferred onto half-strength MS rooting medium supplemented with $6 \mathrm{mg} / \mathrm{L}$ agar, $25 \mathrm{mg} / \mathrm{L}$ sucrose and $300 \mathrm{mg} / \mathrm{L} \mathrm{Cef}$ 
with no added hormones. Plants that were well developed were transferred to a greenhouse for experiments. Transformation efficiencies were calculated using the following equation: (number of transformed explants/total number of explants) $\times 100$.

\subsection{Southern and Northern Blot Analyses}

For southern blot analysis, $10 \mu \mathrm{g}$ of genomic DNA were extracted using the CTAB (cetyl trimethylammonium bromide) method [30,31] from WT plants, three transgenic poplar lines generated using procedures with no improvements to the transformation efficiency (P6-11, P6-17 and P6-23) and three transgenic poplar lines using procedures generated with improvements to transformation efficiency (P6-9, P6-14 and P6-19). The genomic DNA was digested with SalI and EcoRI at $37{ }^{\circ} \mathrm{C}$ for $4 \mathrm{~h}$ and separated overnight on a $0.8 \%$ agarose gel at $15 \mathrm{~V}$. The digested genomic DNA was transferred to a Hybond N+ nylon membrane (Amersham Biosciences BV, Eindhoven, The Netherlands) and detected using DIG (digoxigenin) reagent, according to the manufacturer's instructions (catalog number 11745832910; Roche, Basel, Switzerland). The primers, F5'-TACCGAGGATATTACACTACCAGGA-3' and R 5'-AGTCCAGTTTTGCAGCCAAG-3', were used to generate an 868-bp PCR product to use as a probe on the southern blot. Northern blot analysis was performed using $7 \mu \mathrm{g}$ of total RNA from young leaves of WT plants and three transgenic poplar lines extracted using TRIzol reagent (Tiangen Biotech, Beijing, China), according to the manufacturer's instructions. Total RNA was separated on a $1.2 \%$ agarose formaldehyde gel, transferred to a Hybond $\mathrm{N}+$ nylon membrane (Amersham Biosciences $\mathrm{BV}$ ), and processed for northern hybridization. Random primers labelled with digoxigenin-11-dUTP using DIG-High Prime (Roche) were used to generate probes from the CarNAC6 cDNA (GenBank Accession Number FJ477887.1). The primers, F 5'-CTGGCAAAGGTTGGAGAAAG-3' and R 5'-CGTGTTGGTCTTGTTGTTGT-3', were used to generate a 433-bp PCR product to use as a probe for northern blotting.

\subsection{Statistical Analysis}

All data analyses were performed using ANOVA (analysis of variance) with a mean separation calculated using Duncan's test with SPSS version 16 (SPSS Inc., Chicago, IL, USA) and Excel 2013 software (Microsoft, Redmond, WA, USA). Confidence intervals showing no overlap of the mean values with an error value of 0.05 were considered to indicate statistically significant differences.

\section{Conclusions}

In this study, we demonstrated that the regeneration efficiency of hybrid poplar "Nanlin895" (Populus deltoides $\times P$. euramericana "Nanlin895") could be optimized to yield an $85 \%-90 \%$ regeneration rate. We successfully transformed poplar with the CarNAC6 biotic and abiotic stress resistance gene using Agrobacterium-mediated transformation. We showed that the transformation efficiency was increased by using a two-day preculture of wounded regeneration explants, an Agrobacterium-infective suspension with an $\mathrm{OD}_{600}$ of 0.7 , cocultivation of the Agrobacterium infection suspension with explants for $120 \mathrm{~min}$, an Agrobacterium-infective suspension $\mathrm{pH}$ of 5.0, an AS concentration of $200 \mu \mathrm{M}$, a $4{ }^{\circ} \mathrm{C}$ cold treatment of the Agrobacterium-infective suspension, 
a cocultivation incubation temperature of $28^{\circ} \mathrm{C}$, a cocultivation duration of $72 \mathrm{~h}$ and a cocultivation medium sucrose concentration of $30 \mathrm{~g} / \mathrm{L}$.

\section{Acknowledgments}

We thank Hao Ma (Nanjing Agricultural University, China) for providing the CarNAC6 plasmid and Mohammad Reza Mofid (Isfahan University of Medical Sciences, Iran) and Mahmoud Otroshi (Agricultural Biotechnology Research Institute of Iran, Isfahan) for their technical assistance. We also thank Ehsan Soleymaninejadian for his collaboration in this article. This work was supported by grants from the Forestry Public Benefit Research Program (No. 201304102), the International Science and Technology Cooperation Program of China (2014DFG32440), the National Science Foundation of China (No. 31170561), the Priority Academic Program Development of Jiangsu Higher Education Institutions and the Program for Innovative Research Team at the University of Educational Department and Jiangsu Province, China.

\section{Author Contributions}

Ali Movahedi designed and performed the entire experiments. Qiang Zhuge supervised the research. Jiaxin Zhang and Rasoul Amirian were responsible for data analyses. Ali Movahedi and Qiang Zhuge wrote and edited the manuscript. All authors have read and approved the final manuscript.

\section{Abbreviations}

6-BA, N-6-benzyladenine; AS, acetosyringone; NPTII, neomycin phosphotransferase; ORF, open reading frame; TDZ, thidiazuron; Cef, cefotaxime sodium; Kan, kanamycin sulfate; MS, Murashige and Skoog; Rif, rifampicin; NAA, naphthaleneacetic acid; IAA, indoleacetic acid; IBA, indole-3-butyric acid; NAM, no apical meristem; ATAF1,2, Arabidopsis transcription activation factor; $C U C 2$, cup-shaped cotyledon.

\section{Conflicts of interest}

The authors declare no conflict of interest.

\section{References}

1. Confalonieri, M.; Balestrazzi, A.; Bisoffi, S. Genetic transformation of Populus nigra by Agrobacterium tumefaciens. Plant Cell Rep. 1994, 13, 256-261.

2. Ferreira, S.; Batista, D.; Serrazina, S.; Pais, M.S. Morphogenesis induction and organogenic nodule differentiation in Populus euphratica Oliv. leaf explants. Plant Cell Tissue Organ Cult. 2009, 96, 35-43.

3. Gozukirmizi, N.; Bajrovic, K.; Ipekqi, Z. Genotype differences in direct plant regeneration from stem explants of Populus tremula in Turkey. J. For. Res. 1998, 3, 123-126.

4. Han, X.; Ma, S.; Kong, X.; Takano, T.; Liu, S. Efficient Agrobacterium-mediated transformation of hybrid poplar Populus davidiana Dode $\times$ Populus bollena Lauche. Int. J. Mol. Sci. 2013, 14, 2515-2528. 
5. Kang, B.; Osburn, L.; Kopsell, D.; Tuskan, G.A.; Cheng, Z.M. Micropropagation of Populus trichocarpa "Nisqually-1": The genotype deriving the Populus reference genome. Plant Cell Tissue Organ Cult. 2009, 99, 251-257.

6. Yadav, R.; Arora, P.; Kumar, D.; Katyal, D.; Dilbaghi, N.; Chaudhury, A. High frequency direct plant regeneration from leaf, internode, and root segments of Eastern Cottonwood (Populus deltoides). Plant Biotechnol. Rep. 2009, 3, 175-182.

7. Horsch, R.B.; Fry, J.E.; Hoffmann, N.L.; Eichholtz, D.; Rogers, S.G.; Fraley, R.T. A simple and general method for transferring genes into plants. Science 1985, 227, 1229-1231.

8. Han, K.H.; Meilan, R.; Ma, C.; Strauss, S.H. An Agrobacterium tumefaciens transformation protocol effective on a variety of cottonwood hybrids (genus Populus). Plant Cell Rep. 2000, 19, 315-320.

9. Meilan, R.; Ma, C. Poplar (Populus ssp.). In Methods in Molecular Biology, Agrobacterium Protocols, 2nd ed.; Wang, K., Ed.; Humana Press: Totowa, NJ, USA, 2006; pp. 143-151.

10. Ma, C.P.; Strauss, S.H.; Meilan, R. Agrobacterium-mediated transformation of the genome sequenced poplar clone, Nisqually-1 (Populus trichocarpa). Plant Mol. Biol. Rep. 2004, 22, 1-9.

11. Yevtushenko, D.P.; Santosh, M. Efficient Agrobacterium-mediated transformation of commercial hybrid poplar Populus nigra L. × P. maximowiczii A. Henry. Plant Cell Rep. 2010, 29, 211-221.

12. Husaini, A.M. Pre- and post-agroinfection strategies for efficient leaf disk transformation and regeneration of transgenic strawberry plants. Plant Cell Rep. 2010, 29, 97-110.

13. Bartlett, J.G.; Alves, S.C.; Smedley, M.; Snape, J.W.; Harwood, W.A. High-throughput Agrobacterium-mediated barley transformation. Plant Methods 2008, 4, 1-12.

14. Kondou, Y.; Higuchi, M.; Takahashi, S. Systematic approaches to using the FOX hunting system to identify useful rice genes. Plant J. 2009, 57, 883-894.

15. Nakamura, H.; Hakata, M.; Amano, K.; Miyao, A. A genome-wide gain-of-function analysis of rice genes using the FOX-Hunting system. Plant Mol. Biol. 2007, 65, 357-371.

16. Fowler, S.; Thomashow, M.F. Arabidopsis transcriptome profiling indicates that multiple regulatory pathways are activated during cold acclimation in addition to the CBF cold response pathway. Plant Cell 2002, 14, 1675-1690.

17. Nakashima, K.; Ito, Y.; Yamaguchi-Shinozaki, K. Transcriptional regulatory networks in response to abiotic stresses in Arabidopsis and grasses. Plant Physiol. 2009, 149, 88-95.

18. Nakashima, K.; Takasaki, H.; Mizoi, J.; Shinozaki, K.; Yamaguchi-Shinozaki, K. NAC transcription factors in plant abiotic stress responses. Biochim. Biophys. Acta (BBA) Gene Regul. Mech. 2012, 1819, 97-103.

19. Rabbani, M.A.; Maruyama, K.; Abe, H.; Khan, M.A.; Katsura, K.; Ito, Y.; Yoshiwara, K.; Seki, M.; Shinozaki, K.; Yamaguchi-Shinozaki, K. Monitoring expression profiles of rice genes under cold, drought, and high-salinity stresses and abscisic acid application using cDNA microarray and RNA gel-blot analyses. Plant Physiol. 2003, 133, 1755-1767.

20. Yamaguchi-Shinozaki, K.; Shinozaki, K. Transcriptional regulatory networks in cellular responses and tolerance to dehydration and cold stresses. Annu. Rev. Plant Biol. 2006, 57, 781-803.

21. Holsters, M.; Waele, D.; Depicker, A.; Messens, E.; van Montagu, M.; Schell, J. Transfection and transformation of Agrobacterium tumefaciens. Mol. Gen. Genet. 1978, 163, 181-187. 
22. Tzfira, T.; Frankmen, L.; Vaidya, M.; Citovsky, V. Site-specific integration of Agrobacterium T-DNA via double-stranded intermediates. Plant Physiol. 2003, 133, 1011-1023.

23. Thakur, D.K.; Srivastava, D.K. High-efficiency plant regeneration from leaf explants of male Himalayan poplar (Populus ciliata Wall). In Vitro Cell Dev. Biol. Plant 2006, 42, 144-147.

24. Zhan, X.C.; Kawai, S.; Katayama, Y.; Morohoshi, N. A new approach based on the leaf disc method for Agrobacterium mediated transformation and regeneration of aspen. Plant Sci. 1997, $123,105-112$.

25. Herrera-Estrella, L.; Simpson, J.; Martinez-Trujillo, M. Transgenic plants: An historical perspective. Methods Mol. Biol. 2005, 286, 3-32.

26. Hui, P.; Cheng, H.Y.; Chen, C.; Yu, X.W.; Yang, J.N.; Gao, W.R.; Shi, Q.H.; Zhang, H.; Li, J.G.; $\mathrm{Ma}, \mathrm{H}$. A NAC transcription factor gene of chickpea (Cicer arietinum), CarNAC3, is involved in drought stress response and various developmental processes. J. Plant Physiol. 2009, 166, 1934-1945.

27. Murashige, T.; Skoog, F. A revised medium for rapid growth and bioassays with tobacco tissue culture. Plant Physiol. 1962, 15, 473-497.

28. Horsch, R.B.; Fry, J.; Hoffmann, N.; Neidermeyer, J.; Rogers, S.G.; Fraley, R.T. Leaf disc transformation. In Plant Molecular Biology Manual; Gelvin, S.B., Schilperoort, R.A., Eds.; Kluwer Academic Publishers: Dordrecht, The Netherlands, 1988; pp. 1-9.

29. Satchel, S.E.; Messens, E.; van Montagu, M.; Zambryski, P. Identification of the signal molecules produced by wounded plant cells that activate T-DNA transfer in Agrobacterium tumefaciens. Nature 1985, 318, 624-629.

30. Doyle, J.J.; Dickson, E.E. Preservation of plant samples for DNA restriction endonuclease analysis. Taxon 1987, 36, 715-722.

31. Porebski, L.; Bailey, L.; Baum, B. Modification of a CTAB DNA extraction protocol for plants containing high polysaccharide and polyphenol components. Plant Mol. Biol. Rep. 1997, 15, $8-15$.

(C) 2014 by the authors; licensee MDPI, Basel, Switzerland. This article is an open access article distributed under the terms and conditions of the Creative Commons Attribution license (http://creativecommons.org/licenses/by/3.0/). 